\title{
Das Promotionsstudium in der Rechtswissenschaft
}

\author{
Chancen, Risiken und Perspektiven
}

\author{
Gerrit Hornung/Barbara Sandfuchs"
}

„Promotionsstudium" klingt wie ein Widerspruch in sich - haben Promovierende mit Beginn der Promotion das Studieren und Lernen nicht hinter sich gelassen? „Keineswegs" lautet die Antwort der Befürworterinnen und Befürworter strukturierter Promotionsprogramme. Vielmehr biete die Promotionsphase Gelegenheit, vertiefte methodische und themenspezifische Kenntnisse zu erlangen. Doch wie lässt sich das Konzept eines Promotionsstudiums in der deutschen Rechtswissenschaft umsetzen? Der folgende Beitrag nimmt die fünfte Jahrestagung des Zentrums für rechtswissenschaftliche Fachdidaktik am 18. und 19. März 2014 in Hamburg zum Anlass, dieser Frage nachzugehen.

\section{A. Die juristische Promotion: Attraktivität und neue Wege}

Die Erlangung eines Doktortitels ist in Deutschland für viele Absolventinnen und Absolventen augenscheinlich hochgradig attraktiv: Die Promotionsraten bewegen sich im internationalen Vergleich in der Spitzengruppe. ${ }^{1}$ In der Rechtswissenschaft lag der Anteil der erfolgreichen Promotionen zuletzt bei über 15\% der Absolventinnen und Absolventen; in den Jahren 2007 bis 2009 wurden im Mittel 1.641 Promotionen abgeschlossen. ${ }^{2}$ Ein wesentlicher Grund für die relativ hohen Zahlen dürfte sein, dass der Doktortitel hierzulande nicht ausschließlich als Basis für eine wissenschaftliche Karriere, sondern auch als generell karrierefördernd erachtet wird. Damit korrespondieren meist bevorzugte Einstellung, höhere Gehälter ${ }^{3}$ und bessere Aufstiegschancen.

Die typische deutsche juristische Promotion läuft anders ab als in vielen anderen Disziplinen oder im Ausland, wo vielfach in Anlehnung an das US-amerikanische Modell oder die dritte Phase des gestuften Studiums im Sinne des Bologna-Prozes-

* Der Autor Hornung ist Inhaber des Lehrstuhls für Öffentliches Recht, IT-Recht und Rechtsinformatik an der Universität Passau und Mitglied des dortigen Graduiertenkollegs „Privatheit“ der Deutschen Forschungsgemeinschaft (DFG). Die Autorin Sandfuchs ist Doktorandin am vorgenannten Lehrstuhl und Stipendiatin in diesem DFG-Graduiertenkolleg.

1 Jaksztat/Preßler et al., Promotionen im Fokus, Hannover 2012, S. 3; Konsortium Bundesbericht Wissenschaftlicher Nachwuchs, Bundesbericht Wissenschaftlicher Nachwuchs 2013, Kurzfassung, Bielefeld 2013, S. 10.

2 Wissenschaftsrat, Perspektiven der Rechtswissenschaft in Deutschland, Drs. 2558-12, Hamburg 2012, S. 99; dort wurden die Absolventenzahlen der Jahre 2002 bis 2004 mit den abgeschlossenen Promotionen der Jahre 2007 bis 2009 ins Verhältnis gesetzt.

3 Fünf Jahre nach Abschluss des Universitätsstudiums liegen fächerübergreifend die Gehälter Promovierter jedenfalls in der Privatwirtschaft über denen Nicht-Promovierter; s. Falk/Küpper, Verbessert der Doktortitel die Karrierechancen von Hochschulabsolventen?, Beiträge zur Hochschulforschung 35 (2013), Heft 1, S. 66; Details zu den entsprechenden Nettoeinkommen bei Hauss/Kaulisch et al., Promovierende im Profil. Wege, Strukturen und Rahmenbedingungen von Promotionen, Berlin 2012, S. 42 ff.; zu den Berufsperspektiven auch Konsortium Bundesbericht Wissenschaftlicher Nachwuchs, Bundesbericht Wissenschaftlicher Nachwuchs 2013, Kurzfassung, Bielefeld 2013, S. 19 ff. 
ses $^{4}$ ausgebaute und strukturierte Promotionsstudiengänge angeboten werden. Demgegenüber steckt dieses Konzept in der deutschen Rechtswissenschaft noch in den Kinderschuhen. ${ }^{5}$ Ein universitäts- und fächerübergreifendes Modell existiert bislang nicht, obwohl die ersten strukturierten Promotionsprogramme bereits vor über 25 Jahren starteten. ${ }^{6}$ Die Initiatoren der bisherigen Beispiele - insbesondere der Graduiertenkollegs der Deutschen Forschungsgemeinschaft - müssen daher an ihrer jeweiligen Universität häufig echte Pionierarbeit leisten: Chancen der Finanzierung sind auszuloten, die Rückendeckung der Universität zu sichern, ${ }^{7}$ Organisationsmodelle zu entwickeln und neue Wege zur Rekrutierung der Doktorandinnen und Doktoranden einzuschlagen. ${ }^{8}$

All dies bindet zumindest in der Anfangszeit viel Zeit bei allen Beteiligten. Doch dieser Einsatz lohnt sich: Auch für deutsche Juristinnen und Juristen bietet die strukturierte Promotionsausbildung in ihren verschiedenen Ausprägungen wertvolle Möglichkeiten für den Kompetenzerwerb der Promovierenden, die Forschungsaktivitäten der Betreuenden und die wissenschaftliche Schwerpunktbildung der Universitäten. Voraussetzung ist freilich die Erarbeitung geeigneter Konzepte, die nicht nur die Erfahrungen aus dem Ausland aufnehmen, sondern in Abwägung der Vor- und Nachteile eines intensiven Promotionsstudiums die Interessen aller Beteiligten angemessen widerspiegeln.

Wertvolle Impulse hierzu verspricht die fünfte Jahrestagung des Zentrums für rechtswissenschaftliche Fachdidaktik (ZerF) der Universität Hamburg. Sie wird sich am 18. und 19. März 2014 mit dem Thema „Promotionsstudium in der Rechtswissenschaft" beschäftigen und sowohl Lehrende aus Universitäten und Fachhochschulen als auch Beschäftigte staatlicher Justizprüfungsämter, Promovierende und Studierende adressieren. Die dort erzielten Ergebnisse sollen zur Weiterentwicklung der Promotionsausbildung für Juristinnen und Juristen beitragen. Vor dem Hintergrund der Passauer Erfahrungen des DFG-Graduiertenkollegs „Privatheit“9 und als Anregung für die Diskussionen auf der Tagung werden im Folgenden die in diese Ausbildung gesetzten Hoffnungen und offene Fragen erörtert.

4 Zum Verhältnis der deutschen Reformdiskussion zu diesem Prozess und der teilweise geäußerten Kritik s. den Überblick bei Hauss/Kaulisch et al., Promovierende im Profil. Wege, Strukturen und Rahmenbedingungen von Promotionen, Berlin 2012, S. 35 ff.

5 Im Wintersemester 2010/11 waren sieben Prozent der rechts-, wirtschafts- und sozialwissenschaftlichen Promotionen in Deutschland in strukturierte Programme eingebettet (fächerübergreifender Durchschnitt: acht Prozent), s. Statistisches Bundesamt, Promovierende in Deutschland 2010, Wiesbaden 2012, S. 23.

6 Zu den Hintergründen und der frühen Entwicklung s. z.B. Schuster, WissR 1987, S. 40 ff.; ders., WissR 1989 , S. $253 \mathrm{ff}$.

7 Hierfür gibt es teilweise rechtliche Vorgaben: Anträge auf Graduiertenkollegs beschließt z.B. nach Art. 25 Abs. 3 Nr. 3 Bayerisches Hochschulgesetz der Senat der jeweiligen Universität.

$8 \mathrm{Zu}$ den mit Graduiertenkollegs verbundenen Rechtsfragen s. schon Vierhaus, NVwZ 1992, S. 43 ff.

9 Vgl. http://www.privatheit.uni-passau.de/ (17.12.2013); näher Sandfuchs, JuS 11/2012, S. XL ff. 


\section{B. Bestandsaufnahme und Hoffnungen}

Angehenden Promovierenden bietet sich eine Bandbreite an möglichen Kontexten zur Durchführung der Promotion. Diese Vielfalt ist eine Stärke und soll auch nach dem Willen der Bundesregierung erhalten bleiben. ${ }^{10}$ Ein großer Teil der jungen Wissenschaftlerinnen und Wissenschaftler entscheidet sich dabei traditionell für eine Tätigkeit am Lehrstuhl der Doktormutter beziehungsweise des Doktorvaters. Alternativen bieten externe Promotionen, die durch berufliche Tätigkeiten (vor allem in Kanzleien oder Unternehmen), Stipendien, familiäre Unterstützung oder eigene Mittel finanziert werden. Häufig erscheint die Frage der Finanzierung der Promotion oder, wie vielfach empfunden: die Finanzierung der „freien“ Zeit, die für sie benötigt wird - als entscheidende Weichenstellung. ${ }^{11}$ Darüber kann die Entscheidung in den Hintergrund geraten, wie die Promotionszeit strukturiert werden kann und ob an ihrem Ende noch mehr stehen soll als „nur“ die fertige Arbeit nebst dem erworbenen Titel. Außerdem kommt es bei mehreren der genannten Finanzierungsmodelle vielfach zu Konflikten hinsichtlich der Aufteilung zwischen Arbeits- und Promotionszeit.

Abweichend von den genannten Pfaden kann die Promotion in Anlehnung an internationale Vorbilder im Rahmen eines strukturierten Promotionsprogramms angestrebt werden. Die Möglichkeit hierfür bieten Graduiertenkollegs, Graduiertenschulen, Research Schools oder universitätsinterne Promotionsstudiengänge, wobei Letztere häufig nicht von einem Stipendium begleitet werden. In der Überzahl sind bislang Graduiertenkollegs. Immer mehr Hochschulen fördern ihren wissenschaftlichen Nachwuchs im Rahmen dieser befristeten Einrichtungen. ${ }^{12}$ Die Promovierenden sollen so die Möglichkeit erhalten, ihre Arbeit im Rahmen eines koordinierten, von mehreren Hochschullehrenden getragenen Forschungsprogramms durchzuführen. Die Finanzierung erfolgt häufig durch die Deutsche Forschungsgemeinschaft und die Max-Planck-Gesellschaft, daneben aber auch durch andere außeruniversitäre Forschungseinrichtungen sowie länder- und hochschulbezogene Programme. Schon seit

10 S. dazu Vierter Bericht über die Umsetzung des Bologna-Prozesses in Deutschland, BT-Drucks. 17/8640, S. 8 und 17.

$11 \mathrm{Zu}$ der Verteilung der verschiedenen Finanzierungsmodelle s. Jaksztat/Preßler et al., Promotionen im Fokus, Hannover 2012, S. $15 \mathrm{ff}$.

12 Zur Entwicklung s. Deutsche Forschungsgemeinschaft, 20 Jahre Graduiertenkollegs, Bonn 2010. 
den 1980er Jahren, vermehrt aber in jüngster Zeit gibt es auch eine Reihe entsprechender juristischer Promotionsprogramme. ${ }^{13}$

Interdisziplinäre Arbeit, herausragende akademische Betreuung, ein begleitendes Studienprogramm sowie ein komfortables monatliches Stipendium - so oder ähnlich lesen sich die einschlägigen Ausschreibungen. Frei von zusätzlicher Arbeitsbelastung sollen die Promovierenden an dem begleitenden Ausbildungsprogramm teilnehmen sowie in Vollzeit an ihren jeweiligen Forschungsvorhaben arbeiten. ${ }^{14}$ Dabei werden die strukturierten Promotionsprogramme häufig von mehreren Professuren und teilweise auch von verschiedenen Fakultäten getragen.

Durch intensiven Kontakt, gemeinsam festgelegte Meilensteine, regelmäßige Vorträge über den wissenschaftlichen Fortschritt der eigenen Forschung und eine kontinuierliche Evaluation der Arbeitsschritte soll es möglich sein, die Promotion im anvisierten Zeitraum von zwei bis drei Jahren zum Abschluss zu bringen. Ergänzend werden eine Unterstützung bei eigenen Publikationen, Vorträgen oder Bewerbungen zu Forschungsaufenthalten an international renommierten Universitäten und eine entsprechende Finanzierung geboten. Besonders forciert wird das Einreichen eigener deutsch- wie englischsprachiger Beiträge zu Tagungen.

Zusätzlich zur fachlichen Betreuung durchlaufen die Promovierenden ein begleitendes Studienprogramm, das beispielsweise Vorträge an der eigenen Universität, interdisziplinäre und fachspezifische Seminare oder Soft-Skills-Kurse umfassen kann. Auch werden weitere Schwerpunkte gelegt, beispielsweise auf das Sammeln erster

$13 \mathrm{Zu}$ den ersten Kollegs mit juristischer Beteiligung und ihrer Einbettung in die Entwicklung der rechtswissenschaftlichen Forschung s. z.B. Schmidt-Aßmann, JZ 1995, S. 2 (6). In der Vergangenheit sind etwa - ohne Anspruch auf Vollständigkeit - zu nennen die Graduiertenkollegs „Europäische mittelalterliche Rechtsgeschichte, neuzeitliche Rechtsgeschichte und juristische Zeitgeschichte “ in Frankfurt a.M. (s. Diestelkamp, JuS 1989, S. 942; Rückert, ZEuP 1997, S. 531), „Umwelt- und Technikrecht“ in Trier (s. Preuss, NVwZ 1993, S. 866), „Europäisches Privat- und Wirtschaftsrecht“ in Berlin (s. Flessner, JuS 1997, S. 190), „Internationalisierung des Privatrechts“ in Freiburg (s. Hohloch, ZEuP 1996, S. 703), „Risikoregulierung und Privatrechtssystem“ in Bremen, „Infrastruktur für den elektronischen Markt“ in Darmstadt und „Europäisches und Internationales Wirtschaftsrecht” in Bonn. Aktuelle Beispiele bilden die DFG-Graduiertenkollegs „Geistiges Eigentum und Gemeinfreiheit“ in Bayreuth, „Ökonomik der Internationalisierung des Rechts“ in Hamburg, „Verbesserung von Normsetzung und Normanwendung im integrierten Umweltschutz durch rechts- und naturwissenschaftliche Kooperation“ in Trier, „Verfassung jenseits des Staates“ in Berlin und „Privatheit“ in Passau (s. Fn. 9). Die aktuell durch die DFG geförderten Graduiertenkollegs sind unter http://www.dfg.de/foerderung/programme/listen/index.jsp?id=GRK (17.12.2013) abrufbar.

Die Max-Planck-Gesellschaft bietet derzeit im Bereich Rechtswissenschaften vier Research Schools an, nämlich für „Comparative Criminal Law”, „Retaliation, Mediation and Punishment”, „Successful Dispute Resolution in International Law” und „Comparative Legal History”, s. http://www.mpg.de/ de/imprs (17.12.2013).

Hinzu kommen Graduiertenkollegs anderer Träger (beispielsweise bei den Kollegs „Konstitutionelle Grundlagen globalisierter Finanzmärkte - Stabilität und Wandel“ in Jena und Halle-Wittenberg sowie „Law and Economics of Money and Finance“ in Frankfurt a.M.) sowie weiterer interdisziplinärer Einrichtungen.

14 Während fächerübergreifend Promovierende in strukturierten Promotionsprogrammen täglich durchschnittlich 5,9 Stunden für die Arbeit an der Promotion aufwenden, liegt der Wert bei wissenschaftlichen Mitarbeiterinnen und Mitarbeitern an universitären Lehrstühlen nur bei 3,3 Stunden; s. Jaksztat/Preßler et al., Promotionen im Fokus, Hannover 2012, S. 60. 
Erfahrungen im Lehrbetrieb, auf individuelles Karriere-Coaching oder auch auf das Knüpfen von Kontakten in die Wirtschaft.

Durch diese Zusatzprogramme sollen nicht nur die Arbeit an dem eigenen Forschungsvorhaben erleichtert, sondern auch die Grundlage für die weitere berufliche Entwicklung gelegt werden. Ziel ist es, den Promovierenden nach Abschluss der Promotion einen guten Zugang sowohl zur Wissenschaft als auch zur Wirtschaft zu bieten. Weiter sollen strukturierte Programme helfen, umfassende Kontakte zur nationalen und internationalen Fachwelt zu knüpfen und so Anregungen für eigene Forschungsvorhaben und den weiteren Werdegang zu gewinnen.

\section{Offene Fragen}

Geförderte Promotionsstudiengänge begünstigen (und erfordern) das Nachdenken über die Struktur der Promotion. Sie stellen damit eine Irritation hergebrachter Abläufe dar, die in neue Entwicklungen münden kann - aber auch zur Selbstvergewisserung und Bestätigung der bisherigen Verfahren beizutragen vermag. Strukturierte Promotionsprogramme in der Rechtswissenschaft sind kein Selbstzweck, sondern können nur dann erfolgreich sein, wenn sich alle Beteiligten über die Herausforderungen dieser Promotionsform im Allgemeinen und über die Spezifika des deutschen und speziell juristischen Promotionsstudiums bewusst sind.

\section{Ziele und Zielkonflikte des Promotionsstudiums}

Schon die Ziele strukturierter Promotionsprogramme sind vielschichtig. Zu nennen sind beispielsweise: ${ }^{15}$

- der Erwerb zusätzlicher, über die Promotion hinausgehender wissenschaftlicher und praktischer Qualifikationen durch strukturierte Angebote,

- der Austausch zwischen den Teilnehmenden durch gegenseitiges Feedback und die Betreuung durch Postdoktorandinnen und -doktoranden anstelle der sonst vielfach anzutreffenden „Isolationsarbeit“,

- die durch diesen Austausch sowie eventuell durch fachfremde Zweit- oder Drittbetreuung erreichbare Interdisziplinarität,

- ein engeres und verbindlicheres Verhältnis von Promovierenden und Betreuenden (beispielsweise durch Betreuungsvereinbarungen),

- die Befreiung der Promovierenden aus starken Abhängigkeitsverhältnissen (vor allem an Lehrstühlen mit oftmals überobligatorischer Arbeitsbelastung auf Teilzeitstellen),

- die Clusterbildung durch eine Förderung thematisch verwandter Promotionen an einem universitären Standort sowie die entsprechende Bündelung von Forschungsaktivitäten, die - ebenso wie die Erhöhung der Promotionszahlen - auch der Außendarstellung der Universitäten nützt,

$15 \mathrm{Zu}$ den Zielen, die der Wissenschaftsrat im Jahre 1986 in seinen „Empfehlungen zur Struktur des Studium“ mit dem damals für Deutschland pionierhaften Vorschlag der Einrichtung von Graduiertenkollegs verfolgte, s. Schuster, WissR 1987, S. 40 (42 ff.). 
- die Verstärkung von Verbundforschungen, bei denen Universitäten mit anderen Forschungsinstitutionen sowie gegebenenfalls mit der Forschung in der Wirtschaft zusammenwirken,

- eine Verbesserung der geschlechterspezifischen Gleichstellung, die in strukturierten Programmen leichter erreichbar ist als bei der individuellen Betreuung von Promotionen und der singulären Besetzung von einzelnen Stellen,

- der Ausbau der internationalen Sichtbarkeit durch die Einbindung ausländischer Promovierender, Betreuender und Gäste,

- ein frühzeitiger Wissenstransfer durch die Unterstützung bei ersten Publikationen und die geförderte Teilnahme an Tagungen,

- die Plagiatsvermeidung durch eine intensive begleitende Betreuung mit iterativen Vorträgen über den jeweiligen Zwischenstand

- sowie schließlich die allgemeine und spezifische Fortbildung durch Veranstaltungen, Forschungskolloquien, Workshops und Sommerschulen, an denen auch externe Gäste und Teilnehmende mitwirken können.

Die Bewertung dieser Ziele liegt im Auge des Betrachtenden und wird auch aus seiner Perspektive nicht konfliktfrei sein. So bestehen aus Sicht der Promovierenden Vorund Nachteile, die oftmals mit der persönlichen Arbeitsweise zusammenhängen (beispielsweise: Strukturierung als Unterstützung der eigenen Arbeit oder als belastende und beschränkende Verschulung, zusätzliche Angebote als Möglichkeit zum Kompetenzerwerb oder als zeitliche und ressourcenmäßige Belastung, definierte Zwischenziele und Deadlines als Chance zur Selbstmotivierung oder als Auslöser psychischer Blockaden). Die Ziele der Betreuenden werden möglicherweise weniger in der oftmals zeitintensiven zusätzlichen Betreuung durch regelmäßige Kolloquien und Zwischenbesprechungen, sondern eher in der Nutzung der Graduiertenkollegs für die eigene Forschungstätigkeit, die Verbesserung der Kontakte und die Erhöhung der eigenen Reputation liegen. Auch Fakultäten, Institute und Universitäten verfolgen eigene Interessen, während Geldgeber neben der wissenschaftlichen Exzellenz auch übergeordnete Belange des Wissenschaftsstandorts Deutschland berücksichtigen werden.

Die verschiedenen Interessen und Belange werden teilweise parallel laufen, teilweise aber auch in Konflikt kommen. Eine Aufgabe - und Chance - der Tagung des ZerF sollte es deshalb sein, diese Interessen zusammenzutragen, mögliche Konflikte transparent zu machen und Mechanismen für ihre Austragung aufzuzeigen.

\section{Wissenschafts- oder Praxisorientierung?}

Anders als im internationalen Vergleich dient die Promotion in Deutschland vielfach nicht (nur) als Grundlage einer wissenschaftlichen Karriere, sondern als zusätzliche Qualifikation auf dem juristischen Arbeitsmarkt. Die Erfahrung zeigt, dass Promovierte bei Einstellungsentscheidungen Vorteile haben - Kanzleien und Unternehmen 
honorieren offenbar die unter Beweis gestellte Eignung zur selbstständigen wissenschaftlichen Arbeit, aber auch die Berechtigung zur Führung des Doktortitels. ${ }^{16}$

Vor diesem Hintergrund erscheint es klärungsbedürftig, inwieweit strukturierte Promotionsprogramme neben der wissenschaftlichen Ausbildung auch eine Vorbereitung auf die Praxis leisten können und sollen. Gerade weil die in vielen technischen Fächern üblichen „Industriepromotionen“ in der Rechtswissenschaft kaum möglich sind, könnten praktische Elemente und der Erwerb von Schlüsselqualifikationen einen Beitrag zum viel und kontrovers diskutierten Ziel der „Employability“ leisten. Dadurch würde dem Vorwurf entgegengewirkt, eine Promotion führe zur Praxisuntauglichkeit der Betroffenen. Das offensichtliche Risiko besteht allerdings in der Überfrachtung der Programme, die überdies Interessierte mit wissenschaftlichen Karrierezielen abschrecken könnte.

Für diese Zielgruppe stellt sich außerdem die Frage der Einbindung in Lehr- und Forschungskontexte: Die Durchführung einer Promotion im Rahmen eines strukturierten Promotionsprogramms birgt - in Ermangelung einer promotionsbegleitenden Lehrstuhltätigkeit - für Promovierende das Risiko, keine Lehr- sowie nur eingeschränkte Forschungserfahrung zu sammeln. Soweit das Ziel des strukturierten Promotionsprogramms auch ist, die Promovierenden auf eine wissenschaftliche Tätigkeit vorzubereiten, bedarf es deshalb spezieller Maßnahmen der Einbindung in Lehrund Forschungskontexte.

\section{Promotionsthema, wissenschaftliche Bearbeitung und Prüfungsleistung}

Auswahl, Konkretisierung und endgültige Abgrenzung des Themas sind Kernfragen der Promotion. Durch das gemeinsame Oberthema weisen strukturierte Promotionsprogramme hier Besonderheiten auf. ${ }^{17}$ Es wird deshalb zu erörtern sein, wie eng thematische Festlegungen gehandhabt werden sollen. Zu disparate Themen lassen die Synergieeffekte zwischen den einzelnen Arbeiten abnehmen, zu enge Festlegungen begrenzen die Möglichkeiten der Promovierenden, ihre Forschungen im Laufe der Zeit signifikant neu zu justieren. Wenn man akzeptiert, dass sich im Zuge der Bearbeitung auch wesentliche Teile und Fragestellungen als Sackgassen erweisen können, wird man hier im Interesse der Promovierenden in Einzelfällen nicht umhin kommen, auch Verschiebungen zu ermöglichen, die das Promotionsthema an den Rand des Oberthemas - oder sogar darüber hinaus - bringen.

Zur Unterstützung der wissenschaftlichen Bearbeitung lässt sich in Anlehnung an internationale Modelle und andere Fachdisziplinen fragen, inwieweit eine Integrati-

16 Daneben mag auch die Vermutung einer hohen Punktzahl in mindestens einem Staatsexamen eine Rolle spielen, die häufig Promotionsvoraussetzung ist.

17 Dasselbe gilt für die Promotion im Rahmen von Forschungsprojekten, wo die Themenwahl häufig noch stärker eingeschränkt ist. Wenig überraschend weist die Gruppe der frei Promovierenden die höchste Quote selbst gewählter Promotionsthemen auf: 80\% gegenüber 45\% in strukturierten Programmen und $20 \%$ in Forschungsprojekten; s. Jaksztat/Preßler/etz al., Promotionen im Fokus, Hannover 2012, S. 19. 
on forschungsorientierter Komponenten schon in das Studium sinnvoll ist. ${ }^{18}$ Strukturierte Promotionsprogramme könnten davon profitieren, weil die Entscheidung für oder gegen das Anstreben einer Promotion, die Wahl des Themas, das Anfertigen des vielfach für die Bewerbung erforderlichen Exposés sowie später die Bearbeitung erleichtert würden. Dagegen steht der Nachteil, dass allen Studierenden ohne Rücksicht auf deren angestrebte Karrierewege zusätzliche Belastungen auferlegt würden. $\mathrm{Ob}$ und in welcher Form freiwillige forschungsorientierte Zusatzangebote einen Mittelweg bilden können, bedürfte näherer Untersuchung und Erprobung.

Schließlich stellt sich die Frage der Bewertung: Wenn strukturierte Programme gerade mehr erreichen wollen als Doktorarbeit plus Rigorosum oder Disputation, so könnten diese zusätzlichen Teile separat geprüft und bestätigt werden, ${ }^{19}$ perspektivisch sogar in die Note einfließen oder zumindest die Teilnahme an entsprechenden Angeboten zur Voraussetzung des erfolgreichen Abschlusses aufgewertet werden. ${ }^{20}$ Auch ohne derartige rechtliche Veränderungen müssen sich die Betreuenden die Frage stellen, wie sie im Rahmen der Bewertung damit umgehen, dass sie selbst und weitere Mitglieder der Programme im Rahmen von Kolloquien und Zwischenevaluationen in aller Regel viel mehr Feedback geben werden als bei anderen Promotionsformen. ${ }^{21}$

\section{Betreuungsrahmen und Betreuungsverhältnis}

Neben die Betreuung durch die Doktormutter beziehungsweise den Doktorvater tritt in strukturierten Promotionsprogrammen ein sekundärer Betreuungsrahmen. Dieser wird in einem weiteren Sinne durch alle gebildet, die als Betreuende im jeweiligen Programm mitwirken (beispielsweise auch Postdoktorandinnen und -doktoranden). Vor allem aber werden den Promovierenden typischerweise weitere Hochschullehrende individuell zugeordnet: So werden (fächerübergreifend) in strukturierten Programmen 38 \% der Promovierenden sogar von mehr als drei Personen betreut. ${ }^{22}$

Diese zusätzliche Betreuung über den gesamten Zeitraum der Bearbeitung hinweg (anstelle der reinen ex-post-Einbeziehung im Rahmen der Zweitbegutachtung) ist eine erhebliche Chance für die Promovierenden. Zugleich entsteht jedoch auch eine Belastung für die Betreuenden, beispielsweise in Gestalt wöchentlich stattfindender Kolloquien und individueller Besprechungstermine. Es wird sich die Frage stellen, ob

18 Dazu Frenzel, in: Brockmann/Dietrich et al. (Hrsg.), Methoden des Lernens in der Rechtswissenschaft, Baden-Baden 2012, S. 104 ff.

19 So schon im Graduiertenkolleg „Umwelt- und Technikrecht“ in Trier; s. Preuss, NVwZ 1993, S. 866 (867).

20 Die in Graduiertenkollegs üblichen Betreuungsvereinbarungen enthalten vielfach Selbstverpflichtungen der Promovierenden zum Besuch von Kolloquien, Seminaren oder Workshops. Ohne Regelung in der jeweiligen Promotionsordnung wird dieser Besuch aber nicht zu einer rechtlichen Promotionsvoraussetzung.

21 Ähnliche Fragen stellen sich allerdings schon seit jeher bei Promotionsformen, bei denen gemeinsam veröffentlichte Zwischenergebnisse direkt in die spätere Dissertation einfließen.

22 Jaksztat/Preßler et al., Promotionen im Fokus, Hannover 2012, S. 27. 
Universitäten diesen Aufwand auf das Deputat anrechnen ${ }^{23}$ oder sonst honorieren, ${ }^{24}$ um eine gute Betreuung zu gewährleisten. ${ }^{25}$

Im Sinne einer zielführenden Betreuung und effektiven Zusammenarbeit bedarf es überdies der Prüfung, inwieweit personelle und inhaltliche Überschneidungen zwischen primärem und sekundärem Rahmen gewünscht sind und wie diese gegebenenfalls erreicht werden können. So wird es gerade in interdisziplinären Promotionsprogrammen sinnvoll sein, die Promovierenden zusätzlich in fachspezifische Kolloquien der Hauptbetreuenden einzubinden - auch um der Gefahr einer Abgabe der Betreuungsaufgaben an „das strukturierte Promotionsprogramm“ entgegenzuwirken.

\section{Weitere Fragestellungen}

Je nach Organisation und Thema des Promotionsstudiums werden die einzelnen Programme weitere Fragen aufwerfen. Soweit die Finanzierung - wie häufig - durch Stipendien erfolgt, stellen sich die mit diesen allgemein verbundenen Probleme: insbesondere die fehlende Sozialversicherung (mit dem Erfordernis der privaten Krankenversicherung und der mangelnden Absicherung nach dem Ende der Promotion), aber auch das im Vergleich zu Vollzeitstellen geringere Einkommen. Praktische Probleme können sich auch ergeben, wenn die Promovierenden zwar einerseits Mittel für besondere Aktivitäten zur Verfügung gestellt bekommen, andererseits aber nicht auf die vielfach wertvollen Ressourcen eines Lehrstuhls (nicht zuletzt den Einsatz studentischer Hilfskräfte) zurückgreifen können.

Schwierigkeiten wirft schlussendlich auch die Konkurrenzsituation mit der Lehrstuhlrekrutierung auf: Aus Sicht von Lehrenden kann es erheblich attraktiver sein, exzellente Absolventinnen und Absolventen für das Lehrstuhl-Team zu gewinnen und so im Rahmen der Unterstützung in Forschung und Lehre unmittelbar von ihren Fähigkeiten zu profitieren, anstatt sie durch die Vermittlung in strukturierte Promotionsprogramme an diese zu „verlieren“. ${ }^{26}$ Dies kann zu nicht unerheblichen inneren Widersprüchen bei den Betreuenden oder sogar zur Ablehnung der Mitwirkung an derartigen Programmen führen. Als Kompromiss kommt hier die Assoziierung in

23 Diese Möglichkeit wird für die Leitung von Graduiertenkollegs z.B. eröffnet in $\$ 7$ Abs. 3 Bremische Lehrverpflichtungs- und Lehrnachweisverordnung und $\mathbb{S} 10$ Abs. 2 Satz 1 Saarländische Lehrverpflichtungsverordnung. Im Hauptamt erbrachte „besondere Lehrangebote für Nachwuchswissenschaftler in Graduiertenkollegs" sind nach $\$ 3$ Abs. 1 Satz 1 Bayerische Lehrverpflichtungsverordnung allgemein auf die Lehrverpflichtung anrechenbar.

24 Einige Länder nennen das Engagement in Graduiertenkollegs ausdrücklich als Kriterium für die Gewährung von besonderen Leistungsbezügen; s. z.B. $\$ 4$ Abs. 6 Bayerische Hochschulleistungsbezügeverordnung, $\mathbb{S} 4$ Abs. 7 Bremische Hochschul-Leistungsbezügeverordnung, $\mathbb{S} 4$ Abs. 6 Hochschulleistungsbezügeverordnung des Landes Sachsen-Anhalt, $\mathbb{} 2$ Abs. 4 Satz 1 Nr. 5 a) Hochschul-Leistungsbezügeverordnung für das Land Mecklenburg-Vorpommern und $\mathbb{} 5$ Abs. 5 Hochschul-Leistungsbezügeverordnung für das Land Nordrhein-Westfalen.

25 Dieses Problem wurde schon frühzeitig erkannt; s. Schuster, WissR 1987, S. 40 (49 f.).

26 Ein vergleichbares Problem stellt sich bei der betreuerseitigen Unterstützung von Bewerbungen um Promotionsstipendien der Begabtenförderungswerke. 
Promotionskollegs in Betracht, um den Promovierenden die entsprechenden Möglichkeiten zu eröffnen.

\section{Ausblick}

Es ist absehbar, dass strukturierte Promotionsprogramme in der deutschen Rechtswissenschaft in den nächsten Jahren erheblich an Bedeutung gewinnen werden. Die ZerF-Tagung wird nicht alle der vorgenannten Probleme lösen oder auch nur behandeln können. Sie bietet aber eine hervorragende Gelegenheit, um die bisher verfolgten Konzepte zu sichten, zu vergleichen und Potenziale für bestehende und zukünftige strukturierte Promotionsprogramme aufzuzeigen. Zudem wird sich erweisen, inwieweit das deutsche juristische System von anderen Ländern und Fachgebieten lernen kann - sei es durch Übernahme von Konzepten oder die Kooperation mit ausländischen Wissenschaftsinstitutionen.

Gleichzeitig wird es sich anbieten, die Vor- und Nachteile stärker vereinheitlichter Modelle einerseits, einer gewissen Wegevielfalt andererseits zu diskutieren. Ein übermäßiger Trend zu strukturierten Promotionsprogrammen könnte eine gewisse Sogwirkung entfalten und die Möglichkeiten externer Promotionen beschneiden, die gerade in der Rechtswissenschaft weithin üblich sind - oder umgekehrt sogar eine Chance bieten, externe Promovierende mit einzubinden, wenn man auf die Bedürfnisse der Praxis Rücksicht nimmt. Wie die angestrebte Vielfalt ${ }^{27}$ der rechtswissenschaftlichen Promotionen in Deutschland in Zukunft konkret ausgestaltet werden soll, bleibt eine spannende Aufgabe für die nächsten Jahre. 